\title{
Bewertung der methodischen Qualität von systematischen Übersichtsarbeiten und Metaanalysen: AMSTAR (A Measurement Tool for the Assessment of Multiple Systematic Reviews)
}

\author{
M. Coenen, G. M. Schuetz, \\ M. Dewey
}

Institut

Department of Radiology,

Charité, Berlin

\section{Bibliografie}

Dol http://dx.doi.org/10.1055/

s-0033-1350140

Fortschr Röntgenstr 2013; 185: 937-940 @ Georg Thieme Verlag KG Stuttgart · New York ISSN 1438-9029

\section{Korrespondenzadresse}

\section{Prof. Marc Dewey}

Department of Radiology, Charité - University Hospital Berlin, Humboldt Universität und Freie Universität zu Berlin Charitéplatz 1

10117 Berlin

Tel.: ++49/30/450527296

Fax: ++49/30/450527996

dewey@charite.de
Evidenzbasierte Methoden nehmen einen immer höheren Stellenwert in der Medizin ein. Systematische Übersichtsarbeiten und Metaanalysen sind in der evidenzbasierten Medizin (EbM) von großer Bedeutung, da sie die höchste Stufe der Evidenz darstellen [1]. Systematische Übersichtsarbeiten zeichnen sich durch eine definierte Fragestellung, systematisches Vorgehen in der Literaturrecherche, der Datenextraktion, der qualitativen Bewertung der eingeschlossenen Studien und dem Darstellen der Ergebnisse aus. Eine Metaanalyse ergänzt diese Schritte durch das quantitative Zusammenfassen der Studiendaten [2]. Aufgrund ihres hohen Stellenwerts werden systematische Übersichtsarbeiten vermehrt zurate gezogen, wenn neue Leitlinien erstellt werden [3-7]. Ein weiterer Vorteil von systematischen Übersichtsarbeiten besteht darin, sich mit ihrer Hilfe innerhalb kurzer Zeit einen umfassenden Überblick über den aktuellen Stand der Forschung und die neuesten Ergebnisse zu verschaffen $[8,9]$, dies kann sich ansonsten aufgrund der Flut an publizierten wissenschaftlichen Arbeiten schwierig gestalten [10]. Somit stellen systematische Übersichtsarbeiten für den praktizierenden Arzt eine wichtige Informationsquelle dar. Aufgrund der Anwendung von systematischen Übersichtsarbeiten in den genannten verantwortungsvollen Bereichen ist eine hohe Qualität dieser Arbeiten von enormer Bedeutung.

Für die Bewertung von klinischen Studien, die in systematischen Übersichtsarbeiten oder Metaanalysen aufgenommen werden, gibt es bereits Werkzeuge. Zum Beispiel das weitverbreitete QUADAS-Werkzeug (Quality Assessment of Diagnostic Accuracy Studies included in Systematic Reviews) [11, 12], mit dessen Hilfe die Qualität von diagnostischen Genauigkeitsstudien eingeschätzt werden kann [11]. Das QUADAS-Werkzeug wurde bereits in einem vorherigen Editorial vorgestellt [13], nun möchten wir solch ein Werkzeug für die Beurteilung der methodischen Qualität von systematischen Übersichtsarbeiten und Metaanalysen präsentieren. Während die Einhaltung der ebenfalls bereits vorgestellten PRISMACheckliste ein hohes Maß an Berichtsqualität garantiert [4, 14], kann mit dem AMSTAR-Werkzeug die methodische Qualität, also die Art und Weise der Durchführung einer systematischen Übersichtsarbeit, beurteilt werden [15]. Dieses Editorial möchte somit einen weiteren Beitrag dazu leisten, den Leser über die in der Radiologie wichtigen diagnostischen Studien [16-20] und Metaanalysen zur diagnostischen Genauigkeit zu informieren [4, 13, 21 - 23].

\section{AMSTAR}

\section{$\nabla$}

Im Jahr 2007 veröffentlichten Shea et al. die Publikation „Development of AMSTAR: a measurement tool to assess the methodological quality of systematic reviews“ [15], in der sie das aus 11 Fragen („Items“) zur methodischen Qualität von systematischen Übersichtsarbeiten bestehende AMSTAR-Werkzeug vorstellten. Shea et al. entwickelten AMSTAR aus bereits bestehenden Qualitätsbeurteilungsinstrumenten und fügten neue, durch Expertenmeinung gewonnene Aspekte hinzu. Die Notwendigkeit für die Erstellung eines neuen Werkzeugs ergab sich aus zwei Gründen. Erstens wurden die vorhandenen Werkzeuge aufgrund ihrer Länge und komplizierten Anwendbarkeit zu wenig genutzt [15]. Zweitens gab es neue methodische Erkenntnisse, die in den älteren Werkzeugen noch nicht berücksichtigt worden waren [15].

\section{Entwicklung des AMSTAR-Werkzeugs $\nabla$}

Die Erstellung des neuen Werkzeugs erfolgte in zwei aufeinanderfolgenden Schritten. Zuerst wurde ein Fragenpool aus dem „Overview Quality Assessment Questionnaire“ (OQAQ) [24], der 10 Punkte beinhaltete, und einer von Sacks et al. entworfenen Checkliste [25], die 24 Punkte beinhaltete, gebildet [15]. Dieser Fragenpool wurde noch um drei neuere methodische Entwicklungen berücksichtigende Punkte erweitert [15]. Diese drei Punkte werden an dieser Stelle kurz erläutert. Die erste Frage nach dem Publikationsstatus der Studien bezieht sich auf den Einschluss von grauer, das heißt nicht veröffentlichter, Literatur, wie zum Beispiel Dissertationen, Meeting und Conference Abstracts in die Suche. Mit dem zweiten Punkt, dem Sprachstatus, soll erfasst werden, ob nur englischsprachige oder auch anderssprachige Studien mit eingeschlossen werden. Die dritte und letzte Frage greift die Erfassung des Vorliegens von Publikationsbias auf. Die somit 37 Punkte umfassende Checkliste wurde von 2 Wissenschaftlern an einem Pool von 99 Übersichtsarbeiten zur methodischen Qualitätsbeurteilung angewendet [15]. Die Übersichtsarbeiten bestanden aus 52 Cochrane-Übersichtsarbeiten (www.cochrane.org) und aus einer Datenbank für systematische Übersichtsarbeiten und Metaanalysen von Moher et al. [26]. Nicht englischsprachige Artikel wurden übersetzt und von allen Artikeln wurden die Autoren, Institutionen und das Journal unkenntlich gemacht (Verblindung) [15]. Mithilfe einer explorativen Faktorenanalyse wurden diejenigen Hauptkomponenten, 
die der 37 Punkte umfassenden Checkliste zugrunde liegen, identifiziert und einer Faktorenrotation ausgesetzt [15]. Fragen mit einer geringen Faktorladung (Korrelation der Variablen mit dem Faktor) von <0,50 wurden entfernt [15]. Durch eine Faktorenanalyse ist es möglich, eine größere Anzahl von Variablen auf eine kleinere Anzahl von Variablen zu reduzieren, die zuverlässige Messgrößen darstellen. In einem zweiten Schritt wurde eine 11-köpfige internationale Expertenkommission im Bereich von Qualitätsbewertung und systematischen Übersichtsarbeiten, bestehend aus Klinikern, Methodikern, Epidemiologen und Reviewern gebildet, die mithilfe einer nominalen Gruppentechnik [27] beschlossen, welche Punkte, die bei der Faktoranalyse identifiziert wurden, in den abschließenden Entwurf aufgenommen werden sollten [15]. Die nominale Gruppentechnik erlaubte es den einzelnen Teilnehmern zuerst, ihre Ideen einzeln aufzuschreiben, diese wurden dann der Reihe nach von einem Moderator zusammengetragen („round robin format“) [15]. Anschließend hatte jedes Mitglied die Möglichkeit, seine Bewertung abzugeben, über welche darauf folgend diskutiert wurde [15]. Die Bewertungen der Gruppe wurden statistisch zusammengefasst, um eine kollektive Gruppenbewertung zu erhalten [15]. Eine separate Frage zur Publikationssprache wurde bei der Faktorenanalyse identifiziert, jedoch einigte sich die Gruppe aufgrund des kontroversen Wissenschaftsstands darauf, diesen Punkt unter Publikationsbias mit aufzunehmen [15]. Die einzelnen Fragen wurden beschrieben und eine Vorversion wurde an die gesamte Gruppe für letzte Änderungen verschickt [15]. Die somit ermittelte und endgültige Version von AMSTAR bestand aus einem Fragenkatalog mit 11 Fragen [15].

\section{Validierung des AMSTAR-Werkzeugs \\ $\nabla$}

Noch im gleichen Jahr wurde ein weiterführender Artikel von Shea et al. veröffentlicht, in dem das AMSTAR-Werkzeug extern validiert wurde [28]. Es wurde eine zufriedenstellende Übereinstimmung der Ergebnisse zwischen den bewertenden Personen (Interrater-Reliabilität), Konstruktvalidität (das Werkzeug misst das, was es messen soll) im Vergleich zu einem weiteren Bewertungswerkzeug [29] und Reliabilität des Gesamtscores (Zuverlässigkeit; Stabilität der Ergebnisse bei erneuter Auswertung) festgestellt [28]. Die Reliabilität zeigte sich durch exzellente Cohen's-Kappa- (0,84) und Pearsons-R-Werte (0,96) [28]. 2009 wurden abermals Übereinstimmung, Konstruktvalidität, Reliabilität und Ausführbarkeit des Werkzeugs bemessen und für gut befunden [30]. Drei Fragen wurden in diesem Zuge in ihrer Wortwahl modifiziert [30].

\section{Verwendungsmöglichkeiten für AMSTAR \\ $\nabla$}

Das AMSTAR-Werkzeug [15] bietet dem Leser systematischer Übersichtsarbeiten die Möglichkeit, diese in ihrer methodischen Qualität, d. h. der Art ihrer Durchführung, zu bewerten und somit die Validität der Ergebnisse der Arbeit besser abschätzen zu können. Die 11 Fragen können mit „Ja“, „Nein“, „Kann nicht beantwortet werden“ oder „Nicht anwendbar“ beantwortet werden [15]. „Kann nicht beantwortet werden“ wird gewählt, wenn der Punkt zwar relevant ist, aber vom Autor nicht beschrieben wurde [30]. „Nicht anwendbar“ wird gewählt, wenn der Punkt nicht relevant ist, z. B. wenn das Poolen von Daten abgefragt wird, was in der entsprechenden Arbeit jedoch nicht durchgeführt wurde [30].
Das AMSTAR-Werkzeug [15] fragt die einzelnen Arbeitsschritte zur Erstellung einer systematischen Übersichtsarbeit detailliert $\mathrm{ab}$, bei denen es, je nach Durchführung, zu Verzerrungen (Bias) in der Aussage der systematischen Übersichtsarbeit kommen kann. Zum Beispiel durch den Ausschluss von grauer Literatur bei dem Festlegen von Einschlusskriterien. Die Fragen sind mit der Zeit durch Notizen ergänzt worden, die dem Leser eine Orientierung zur Punktevergabe geben (http://amstar.ca/Amstar_Checklist.php). Das Werkzeug kann dem Autor dazu dienen, methodische Fehler zu vermeiden, oder dem Leser helfen, diese aufzudecken. Wir haben die originalen 11 AMSTAR-Fragen in deutscher Übersetzung und inklusive erklärender Bemerkungen in der $\bullet$ Tab. 1 zusammengefasst. Im Gegensatz zu anderen Beurteilungswerkzeugen, bei denen ein Summenscore nicht erwünscht ist [31], kann dieser bei AMSTAR erhoben werden und der Gesamtabschätzung einer jeden Übersichtsarbeit dienen [30]. Die Antwortmöglichkeit „Ja“ wird mit einem Punkt bewertet, alle anderen Antwortmöglichkeiten werden mit 0 Punkten bewertet. Der Gesamtscore errechnet sich durch die Addition der vergebenen Punkte [30]. Allerdings ist es ausschließlich Metaanalysen möglich, die Gesamtpunktzahl zu erlangen [30], da die Frage 9 das Poolen von Daten betrifft, welches in systematischen Übersichtsarbeiten nicht angewendet wird. Für systematische Übersichtsarbeiten muss in dieser Frage „Nicht anwendbar“ angegeben werden. Deswegen können nur Metaanalysen alle 11 Punkte erreichen.

Abschließend möchten wir drei Fragen des AMSTAR-Werkzeugs, die unserer Meinung nach einen besonders wichtigen Stellenwert haben, beispielhaft näher ausführen.

\section{Beispielerläuterungen zu 3 ausgewählten Fragen des AMSTAR-Werkzeugs \\ $\nabla$}

\section{Zu Frage 1: Wurde ein 'a priori' Design benutzt?}

In den Notizen der Autoren wird verlangt, dass ein Protokoll, eine Einwilligung der Ethikkommission oder vorab publizierte Forschungsziele vorliegen müssen. Das ‘a priori’ Design ermöglicht dem Leser, nachzuvollziehen, ob Veränderungen des Protokolls erfolgten. Die Transparenz und Qualität der systematischen Übersichtsarbeiten sollen dadurch erhöht werden. Allerdings stellen immer noch wenige Autoren solch ein Protokoll zur Verfügung [32]. Bei systematischen Übersichtsarbeiten für die Cochrane Library erscheint ein Protokoll mit Titel vorab (www.cochrane. de/de/systematische-uebersichtsarbeiten\#protocol). Eine weitere Möglichkeit ist die Registrierung einer systematischen Übersichtsarbeit bei PROSPERO (www.crd.york.ac.uk/PROSPERO/).

\section{Zu Frage 9: War die angewandte Methodik zur} Zusammenfassung der Studienergebnisse adäquat?

Die in einer Metaanalyse eingeschlossenen Studien können sich in Bezug auf ihre Patientenkollektive, Interventionen und Endpunkte (klinische Heterogenität) als auch das Studiendesign und die Qualität (methodische Heterogenität) unterscheiden [23]. Um dies zu testen, sollten die Studien z. B. mithilfe des I²-Tests statistisch auf Heterogenität überprüft werden [33, 34]. Im Unterschied zum Cochran's Q ist $\mathrm{I}^{2}$ unabhängig von der Studienanzahl. Abhängig vom Ergebnis der Heterogenitätsprüfung (Werte $<25 \%$ werden als niedrige, $<50 \%$ als moderate und $>75 \%$ als hohe Heterogenität angesehen) sollte das passende statistische Modell zum Kombinieren der Studienergebnisse ausgewählt werden. Bei homogener Studienlage kann ein Fixed-Effects-Modell verwendet werden, bei 
Tab. 1 AMSTAR - Werkzeug zur Bewertung der methodischen Qualität von systematischen Übersichtsarbeiten (aus dem Englischen übersetzt, Originaltext siehe http://amstar.ca/Amstar_Checklist.php).

\section{Nr. AMSTAR-Frage \\ 1. Wurde ein 'a priori' Design benutzt? \\ Die wissenschaftliche Fragestellung und die Einschlusskriterien sollten festgelegt worden sein, bevor die Übersichtsarbeit durchgeführt wurde. \\ 2. Gab es eine zweifache Studienauswahl und Datenextraktion? \\ Es sollten mindestens zwei unabhängige Auswerter die Datenextraktion vollzogen haben und eine Konsensusprozedur zur Klärung von Unstimmigkeiten sollte erfolgt sein. \\ 3. Wurde eine ausführliche Literatursuche durchgeführt? \\ Mindestens zwei elektronische Datenbanken sollten durchsucht worden sein. Die Arbeit muss den abgedeckten Suchzeitraum und die Datenbanken, die durchsucht wurden, enthalten (z. B. Central, EMBASE und MEDLINE). Schlüsselwörter und/oder MeSH-Begriffe müssen angegeben und, soweit durchführbar, sollte die Suchstrategie zur Verfügung gestellt worden sein. Alle Su- chen sollten durch aktuelle Quellen, Übersichtsarbeiten, Lehrbücher, spezialisierte Register oder das Befragen von Experten auf dem spezifischen Gebiet und durch die Sichtung der Referenzlis- ten der gefundenen Studien ergänzt worden sein.}

4. Wurde der Publikationsstatus als Einschlusskriterium benutzt (Stichwort: graue Literatur)? Die Autoren sollten angeben, dass sie nach Berichten unabhängig vom Publikationstyp gesucht haben. Die Autoren sollten angeben, ob unpublizierte Primärarbeiten und welche Sprachen aus der systematischen Übersichtsarbeit ausgeschlossen wurden.

5. Wurde eine Liste mit ein- und ausgeschlossenen Studien zur Verfügung gestellt? Eine Liste von ein- und ausgeschlossenen Studien sollte verfügbar sein.

6. Wurden die Charakteristika der eingeschlossenen Studien zur Verfügung gestellt? Die Originaldaten der Studien bezüglich Studienteilnehmer, -interventionen und -ergebnisse sollten in aggregierter Form, z. B. einer Tabelle, zur Verfügung gestellt worden sein. Die Spannweite der Charakteristika, z. B. Alter, ethnische Herkunft, Geschlecht, relevante sozioökonomische Daten, Krankheitsstatus, -dauer, -schweregrad oder andere Erkrankungen, aller analysierten Studien sollten angegeben worden sein.

7. Wurde die wissenschaftliche Qualität der Studien bewertet und dokumentiert? 'A priori' Bewertungsmethoden sollten angegeben worden sein (z. B. für Effektivitätsstudien, wenn der Autor sich entschieden hat, nur randomisierte, doppelblinde, placebokontrollierte Studien einzuschließen oder eine verborgene Zuweisung der Probanden als Einschlusskriterium gewählt worden ist); für andere Studientypen sind alternative Aspekte relevant.

8. Wurde die wissenschaftliche Qualität der eingeschlossenen Studien bei der Formulierung der Schlussfolgerungen angemessen berücksichtigt?

Die Ergebnisse der methodischen Strenge und wissenschaftlichen Qualität sollten in der Analyse und den Schlussfolgerungen der Übersichtsarbeit berücksichtigt worden und ausdrücklich beim Formulieren von Empfehlungen erwähnt worden sein.

9. War die angewandte Methodik zur Zusammenfassung der Studienergebnisse adäquat? Für die zusammengefassten Ergebnisse sollte ein Test durchgeführt worden sein, um sicherzustellen, dass die Studien kombinierbar waren und um ihre Homogenität zu beurteilen (Chi-Quadrat-Homogenitätstest, $\mathrm{I}^{2}$ ). Falls Heterogenität vorliegt, ist ein Random-Effects-Modell anzuwenden und/oder die klinische Angemessenheit des Zusammenfassens ist zu berücksichtigen (Ist es überhaupt sinnvoll, zusammen zu fassen?).

10. Wurde die Wahrscheinlichkeit für das Vorliegen von Publikationsbias beurteilt? Eine Beurteilung von Publikationsbias sollte aus einer Kombination von grafischen Hilfsmitteln (z. B. Funnel Plot) und statistischem Test (z. B. Regressionstest nach Egger) bestehen.

11. Wurde angegeben, ob ein Interessenkonflikt vorlag?

Potenzielle Quellen der Unterstützung sollten sowohl in der systematischen Übersichtsarbeit als auch in den eingeschlossenen Studien eindeutig angegeben worden sein.

\section{Bemerkung}

Vermeidung einer nachträglichen Anpassung und dem Vorenthalten von Daten.

Stellt sicher, dass die Studienauswahl und Datenextraktion objektivierbar ist.

Die gefundene Literatur kann je nach Suchstrategie und Ihrer Ausführlichkeit stark variieren. Die Suche sollte für den Leser reproduzierbar sein. Eine Anleitung für die Dokumentation einer Suchstrategie wird demnächst publiziert [39].

Der Einschluss von unveröffentlichter Literatur hat einen maßgeblichen Einfluss auf die Ergebnisse von systematischen Übersichtsarbeiten [40,41].

Den Anmerkungen der Autoren nach sollten die ausgeschlossenen Studien zumindest referenziert sein. Der Leser sollte einen Überblick über die Studien erhalten und die Ergebnisse der Arbeit im Kontext der eingeschlossenen Patienten (z. B. Prävalenz, Alter der Patienten) betrachten können. Diese Faktoren können die Ergebnisse beeinflussen.

Die Qualität der eingeschlossenen Studien kann mithilfe von Beurteilungswerkzeugen bewertet werden, wie z. B. mit QUADAS für diagnostische Genauigkeitsstudien $[11,12]$. Es sollte angegeben werden, wie jede einzelne Studie abgeschnitten hat. Die Qualität der Studien beeinflusst die Validität der Studienergebnisse maßgeblich. Aus diesem Grund ist die Qualität der eingeschlossen Studien auch mitbestimmend für die Ergebnisse der systematischen Übersichtsarbeit.

siehe Text

siehe Text

Bekelman et al. [42] zeigten einen direkten Zusammenhang zwischen durch pharmazeutische Firmen finanzierte Studien und deren Ergebnissen. Finanzierung kann folglich die Ergebnisse von Artikeln beeinflussen. vorliegender Heterogenität muss diese berücksichtigt und es sollte ein Random-Effects-Modell angewandt werden [35, 36].

\section{Zu Frage 10: Wurde die Wahrscheinlichkeit für das Vorliegen von Publikationsbias beurteilt?}

Diese sollte aus einer Kombination von grafischen Hilfsmitteln (z. B. Funnel Plot [37]) und statistischem Test (z. B. Regressionstest nach Egger [37]) bestehen [15]. Publikationsbias beschreibt den Effekt, dass tendenziell vor allem größere Studien mit signifikanten Ergebnissen publiziert werden. Wenn nun ausschließlich diese in die Metaanalyse aufgenommen würden, wäre das Gesamtergebnis verzerrt, das heißt die Effektgröße würde überschätzt [38]. Ob Publikationsbias vorhanden ist, kann mithilfe von unterschiedlichen Methoden überprüft werden. Bei einem Funnel Plot wird auf der X-Achse die Effektgröße gegen die Studiengröße auf der Y-Achse aufgetragen. Eine Asymmetrie des Funnel Plots weist daraufhin, dass Publikationsbias vorliegt. Auf der statistischen Ebene kann die Anwesenheit von Publikationsbias z. B. mit dem Egger's-Test überprüft werden [37].

\section{Schlussfolgerung}

Das AMSTAR-Werkzeug [15] bietet dem Leser eine übersichtliche und sinnvoll strukturierte Möglichkeit, die Qualität einer systematischen Übersichtsarbeit zu bewerten und somit die vorlie- 
genden Ergebnisse in ihrer Relevanz zu beurteilen. Autoren systematischer Übersichtsarbeiten können sich bei der Erstellung an die einzelnen Punkte von AMSTAR halten und somit eine solide methodische Qualität der eigenen Arbeiten gewährleisten.

\section{Widmung}

$\nabla$

Dieses Manuskript ist Professor Bernd Hamm zum 60. Geburtstag gewidmet.

\section{Literatur}

1 Puig S, Felder-Puig R. Evidenzbasierte Radiologie: Ein neuer Ansatz zur Bewertung von klinisch angewandter radiologischer Diagnostik und Therapie. Fortschr Röntgenstr 2006; 178: 671 -679

2 Doria AS. Meta-Analysis and Structured Literature Review in Radiology. Academic Radiology 2005; 12: 399-408

3 Moher D, Cook DJ, Eastwood S et al. Improving the quality of reports of meta-analyses of randomised controlled trials: the QUOROM statement. Quality of Reporting of Meta-analyses. Lancet 1999; 354: 1896-1900

4 Walther S, Schuetz GM, Hamm B et al. Berichtsqualität systematischer Reviews und Metaanalysen: PRISMA (Preferred Reporting Items for Systematic reviews and Meta-Analyses). Fortschr Röntgenstr 2011; 183: $1106-1110$

5 Hergan K, Globits S, Loewe C et al. Aktueller Stellenwert der MSCTA in der Koronargefäßdiagnostik (2011): Klinischer Leitfaden der Österreichischen Gesellschaften für Kardiologie und Radiologie. Fortschr Röntgenstr 2011; 183: 964-971

6 Schreyer AG, Ludwig D, Koletzko S et al. Aktualisierte S 3-Leitlinie zur Diagnostik des Morbus Crohn - radiologische Untersuchungstechniken. Fortschr Röntgenstr 2010; 182: 116-121

7 Rogalla P, Janka R, Baum U et al. CT-Kolografie: Leitlinie der Arbeitsgemeinschaft Gastrointestinale Diagnostik der Deutschen Röntgengesellschaft zur Indikation und technischen Durchführung der endoluminalen Dickdarmdiagnostik mittels Computertomografie (sog. virtuelle Koloskopie). Fortschr Röntgenstr 2008; 180: 466 - 469

8 Staudt M, Krageloh-Mann I, Grodd W. Die normale Myelinisierung des kindlichen Gehirns in der MRT - eine Metaanalyse. Fortschr Röntgenstr 2000; 172: 802-811

9 Rominger M, Wisgickl C, Timmesfeld N. Mikroverkalkungstypen der Brust zur Stratifizierung des Malignitätsrisikos: systematischer Review und Metaanalyse von 10665 Fällen unter besonderer Berücksichtigung von rund-/punktförmigem Mikroverkalkungen. Breast Fortschr Röntgenstr 2012; 184 (12): $1144-1152$

10 Davidoff F, Haynes B, Sackett D et al. Evidence based medicine. BMJ 1995; 310: $1085-1086$

11 Whiting P, Rutjes AW, Reitsma JB et al. The development of QUADAS: a tool for the quality assessment of studies of diagnostic accuracy included in systematic reviews. BMC Med Res Methodol 2003; 3: 25

12 Whiting PF, Rutjes AW, Westwood ME et al. QUADAS-2: a revised tool for the quality assessment of diagnostic accuracy studies. Ann Intern Med 2011; 155: 529-536

13 Schuetz GM, Tackmann R, Hamm B et al. Qualität diagnostischer Genauigkeitsstudien: QUADAS (Quality Assessment of Diagnostic Accuracy Studies included in Systematic Reviews). Fortschr Röntgenstr 2010; 182: $939-942$

14 Liberati A, Altman DG, Tetzlaff J et al. The PRISMA statement for reporting systematic reviews and meta-analyses of studies that evaluate health care interventions: explanation and elaboration. Ann Intern Med 2009; 151: W65-W94

15 Shea BJ, Grimshaw JM, Wells GA et al. Development of AMSTAR: a measurement tool to assess the methodological quality of systematic reviews. BMC Med Res Methodol 2007; 7: 10

16 Ohlmann-Knafo S, Kirschbaum M, Fenzl G et al. Diagnostischer Stellenwert der Ganzkörper-MRT und der Skelettszintigrafie in der ossären Metastasendetektion bei Mammakarzinompatientinnen - eine prospektive Doppelblindstudie an zwei Klinikzentren. Fortschr Röntgenstr 2009; 181: 255-263
17 Heusner TA, Hamami ME, Ertle J et al. Stellenwert der Rotationsangiographie für die Detektion extrahepatischer Shunts vor Radioembolisation. Fortschr Röntgenstr 2010; 182: 603 -608

18 Dietzel M, Zoubi R, Burmeister HP et al. Kombiniertes Staging mittels MR-Mammografie: Evaluation eines erweiterten Protokolls zum Screening nach Fernmetastasen des primären Mammakarzinoms klinische Anwendung und diagnostische Genauigkeit in einer initialen prospektiven Studie. Fortschr Röntgenstr 2012; 184: 618-623

19 Steinwender G, Szolar D, Preidler K et al. Diagnostische Wertigkeit der kontrastverstärkten MSCT-Koronarangiografie bei Patienten mit hochgradiger Koronarverkalkung im klinischen Alltag. Fortschr Röntgenstr 2011; 183: $1145-1150$

20 Weber C, Jensen F, Wedegartner $U$ et al. Klinische Relevanz der Computertomographie unter Notdienstbedingungen - Diagnostische Treffsicherheit, therapeutische Konsequenzen. Fortschr Röntgenstr 2004; 176: $37-47$

21 Tackmann R, Schuetz G, Hamm B et al. Berichtsqualität diagnostischer klinischer Studien: STARD (Standards for the Reporting of Diagnostic accuracy studies). Fortschr Röntgenstr 2010; 182: 655-659

22 Panner J, Schuetz GM, Hamm B et al. Eine systematische Anleitung zum Lesen und Interpretieren diagnostischer Genauigkeitsstudien. Fortschr Röntgenstr 2011; 183: 909-912

23 Schueler S, Schuetz GM, Hamm B et al. Lesen und Interpretieren von Metaanalysen diagnostischer Genauigkeitsstudien. Fortschr Röntgenstr 2011; 183: 799-803

24 Shea $B D C$, Moher $D$, (ed.). Assessing the quality of reports of systematic reviews: the QUOROM statement compared to other tools. In: Systematic Reviews in Health Care: Meta-analysis in context 2001

25 Sacks HS, Berrier J, Reitman D et al. Meta-analyses of randomized controlled trials. N Engl J Med 1987; 316: 450-455

26 Moher D, Cook DJ, Jadad AR et al. Assessing the quality of reports of randomised trials: implications for the conduct of meta-analyses. Health Technol Assess 1999; 3: i-iv, 1-98

27 Harvey $N$, Holmes CA. Nominal group technique: an effective method for obtaining group consensus. Int J Nurs Pract 2012; 18: 188-194

28 Shea BJ, Bouter LM, Peterson J et al. External validation of a measurement tool to assess systematic reviews (AMSTAR). PLoS One 2007; 2: e1350

29 Oxman $A D$, Guyatt GH. Validation of an index of the quality of review articles. J Clin Epidemiol 1991; 44: 1271-1278

30 Shea BJ, Hamel C, Wells GA et al. AMSTAR is a reliable and valid measurement tool to assess the methodological quality of systematic reviews. J Clin Epidemiol 2009; 62: 1013-1020

31 Whiting $P$, Harbord R, Kleijnen J. No role for quality scores in systematic reviews of diagnostic accuracy studies. BMC Med Res Methodol 2005; 5: 19

32 Moher D, Tetzlaff J, Tricco AC et al. Epidemiology and reporting characteristics of systematic reviews. PLoS Med 2007; 4: e78

33 Higgins JP, Thompson SG. Quantifying heterogeneity in a meta-analysis. Stat Med 2002; 21: 1539-1558

34 Higgins JP, Thompson SG, Deeks JJ et al. Measuring inconsistency in meta-analyses. BMJ 2003; 327: 557-560

35 Berkey CS, Hoaglin DC, Antczak-Bouckoms A et al. Meta-analysis of multiple outcomes by regression with random effects. Stat Med 1998; 17: $2537-2550$

36 DerSimonian R, Laird N. Meta-analysis in clinical trials. Control Clin Trials 1986; 7: $177-188$

37 Egger M, Davey SG, Schneider $M$ et al. Bias in meta-analysis detected by a simple, graphical test. BMJ 1997; 315: 629-634

38 Guyatt GH, Oxman AD, Montori V et al. GRADE guidelines: 5. Rating the quality of evidence-publication bias. J Clin Epidemiol 2011; 64: 1277 1282

39 Kable AK, Pich J, Maslin-Prothero SE. A structured approach to documenting a search strategy for publication: A 12 step guideline for authors. Nurse Educ Today 2012

40 Hopewell S, McDonald S, Clarke M et al. Grey literature in meta-analyses of randomized trials of health care interventions.. Cochrane Database Syst Rev 2007,: MR000010

41 McAuley L, Pham B, Tugwell P et al. Does the inclusion of grey literature influence estimates of intervention effectiveness reported in metaanalyses? Lancet 2000; 356: 1228 - 1231

42 Bekelman JE, Li Y, Gross CP. Scope and impact of financial conflicts of interest in biomedical research: a systematic review. JAMA 2003; 289: $454-465$ 